\title{
Nulling interferometry : Lommel's integrals applied to a Fresnel's diffraction effect
}

\author{
Yves Rabbia $^{1} \dagger$, Jean Gay ${ }^{1}$, Bruno Chazelas ${ }^{2}$, Alain Labèque ${ }^{2}$, \\ and Jean-Pierre Rivet ${ }^{1}$ \\ ${ }^{1}$ Observatoire de la Côte d'Azur, Av Copernic, 06130 Grasse, France \\ email: rabbia@obs-azur.fr \\ ${ }^{2}$ Institut d'Astrophysique Spatiale, 91 Orsay, France
}

\begin{abstract}
Nulling Interferometry applied to the search and characterization of earth-like exoplanets requires to eliminate the star's contribution at a rejection level (Rej $=$ collected energy/residual energy)larger than $10^{6}$ over a large bandwidth (6 to $18 \mu \mathrm{m}$ ). Nulling test-benches are in development in several laboratories so as to master such high a rejection. One approach relies on a Mach-Zehnder set-up with Achromatic Phase Shifters (APS). One APS concept is based on the focus-crossing property, providing an intrinsically achromatic phase shift by $\pi$. Using a confocal configuration for the focus-crossing approach, a Fresnel's diffraction effect degrades the rejection. Usual optical engineering softwares fail in assessing rejection performance and an analytical approach is needed. We describe the bench optical configuration and the Fresnel's diffraction effect as well as a possible way for correction. Then we describe the analytical method, based on Lommel's integrals, to evaluate the expectable rejection.
\end{abstract}

Keywords. Nulling interferometry, Exo-Earths, Fresnel's diffraction, Lommel's integrals

\section{Introduction}

Nulling interferometry, recognized as the relevant tool in the search and spectral analysis for earth-like exoplanets, is the baseline of the ESA-Darwin mission (ESA, 2000). In the framework of preliminary studies, nulling test benches are in development so as to master the rejection process, a key-issue for such a mission. A convenient system is a Mach-Zehnder-like interferometer where Achromatic Phase Shifters are installed so as to provide the required $\pi$-phase shift yielding the elimination of the starlight via destructive interferences. Among other approaches (Rabbia et al, 2002) the confocal APS based on focus property (Gouy, 1890) is considered. With this peculiar device a Fresnel's diffraction adverse effect degrade the rejection : Rej $=$ (collected energy)/(residual energy). Eventhough this effect occurs on the bench, not in the flying device, expectable rejection performance on the bench must be calculated. At the very high level of rejection targeted, currently available Optical Engineering Softwares fail to provide reliable results (prohibitively large workspace and sampling rate needed). An analytical approach, using Lommel's formalism (Born \& Wolf, 1980) taking advantage of circular apertures and Gaussian beams, yields an alternate approach for calculation of rejection. We describe the generic set-up of the bench, the APS based on the focus-crossing property, the adverse effect and the analytical method for calculation of rejection. Finally expected rejection versus wavelength curves in the effective conditions of operation on the bench are given.

$\dagger$ Present address: Observatoire de la Cote d'Azur, Dpt Gemini, UMR CNRS 6203, Av Copernic 06130, Grasse -France 


\section{2. generic design, focus-crossing and associated APS}

The set-up basically is a Mach-Zehnder interferometer, fed by a collimated beam from a monomode fiber used as source. The output common beam after recombination by the exit beam splitter is focused on a monomode fiber used as spatial filter.
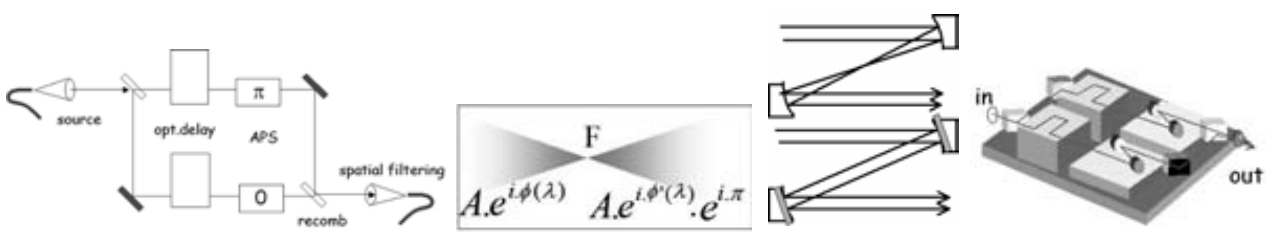

Figure 1. from left to right : principle of mach-zehnder set-up used as nulling test-bench, illustration of the focus-crossing property, principle of the associated APS and global architecture of bench.

In each arm is set an APS component. One component (CF), based on the focuscrossing property, is a confocal assembly of two half-paraboloids. The other component $(\mathrm{FF})$, simply is a couple of flat mirrors set to produce the same optical path and same average incidence as in the other arm.

\section{Adverse effect from Fresnel's diffraction and remedies}

As seen from the recombination plate at output, there is an axial separation for the images of the input pupilla: the CF unit preserve the location of the input pupilla but not the FF unit. Therefore, because of Fresnel's diffraction effect (caused by unequal propagation length in $\mathrm{CF}$ and FF channels) the amplitude distributions set to interfere are not identical what is detrimental to the efficiency of destructive interference, hence to rejection.
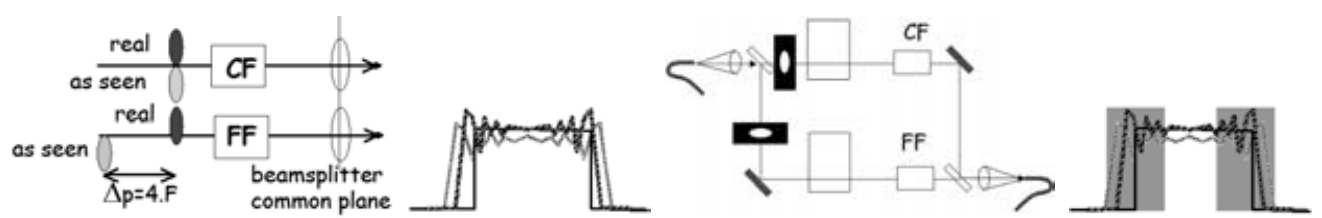

Figure 2. from left : axial separation ( $\mathrm{F}$ : focal length in $\mathrm{CF}$ ) for images of input pupilla on each interferometer arm as seen from recombining plate, illustration of resulting wavefront mismatch, insertion of "corrective" stops, "cleaning" effect of stops.

A way to reduce the degradation is to insert aperture stops at different locations in such way that effective pupilla are now seen in the same plane from the recombination plate. Moreover the diameter of stops is chosen so that amplitude distorsions (mainly peripheral) are significantly eliminated (this, at the price of loosing some photons, rather negligible with gaussian beams).

\section{Using Lommel's formalism}

With a targeted rejection larger than $10^{6}$ wavefronts errors as small as $2.10^{-3}$ radian induce degradation. Softwares fail providing reliable amplitude maps. Analytical derivation of rejection is needed. Since apertures are circular and input wave is Gaussian, Lommel's integrals provide an analytical approach with sufficient accuracy. 


\section{1. propagated complex amplitude}

Notations are in Figure 3 below. Starting from the complex amplitude $A_{0}$ (Gaussian amplitude of width "b" at 1/e) coming out of a circular aperture of radius $\mathrm{R}$, the complex amplitude $A_{p}$ obtained after propagation along the length "p" is given by the usual convolution $A_{p}(\vec{r})=\oint_{r, g^{\prime}<R, \theta} A_{0}(\vec{r}) \cdot Z_{p}\left(\vec{r}-\overrightarrow{r^{\prime}}\right) \cdot r^{\prime} \cdot d r^{\prime} \cdot d \theta$ where we use the usual "propagation operator" $Z_{p}(\vec{r})=\exp \left(i \cdot \frac{2 \cdot \pi \cdot p}{\lambda}\right) \cdot \exp \left(i \cdot \frac{\pi \cdot r^{2}}{\lambda \cdot p}\right)$ circularly symmetric.
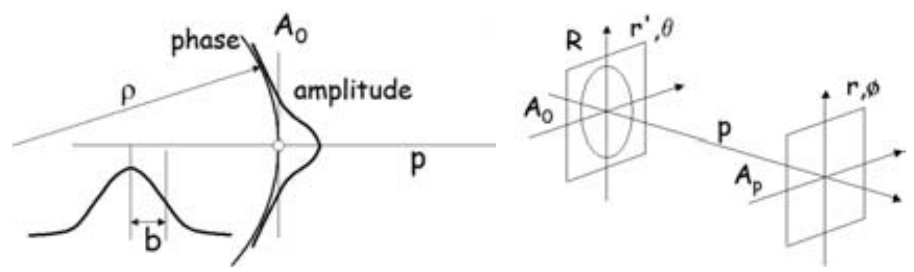

Figure 3. beam and propagation parameters for complex amplitude calculation.

Using a reduced variable $z=r^{\prime} / R$ and after some algebraic work, we set $v=\frac{2 . \pi \cdot r . R}{\lambda \cdot p}$ and $u=2 . \pi \cdot\left[\frac{R^{2}}{\lambda \cdot p}+\frac{R^{2}}{\lambda \cdot \rho}+\frac{i \cdot R^{2}}{b^{2}}\right]$ so that we have the general expression:

$$
A_{p}(\vec{r})=\left[2 \cdot \pi \cdot R^{2} \cdot \frac{A_{0}}{i \cdot \lambda \cdot p} \cdot \exp \left(i \cdot \frac{2 \cdot \pi \cdot r^{2}}{\lambda \cdot p}\right)\right] \cdot I[u, v]
$$

with $I[u, v]=\int_{0}^{1} J_{0}(z \cdot v) \cdot \exp \left(\frac{i}{2} \cdot z^{2} \cdot u\right) \cdot z \cdot d z$

Depending on the value of the set-up parameters included in " $\mathrm{u}$ " and "v", we have:

$I[u, v]=\frac{i}{u} \cdot \exp \left(\frac{i}{2} \cdot u\right) \cdot\left[\sum_{n=1}^{\infty}\left(\frac{u}{i \cdot v}\right)^{n} \cdot J_{n}(v)\right]$ when $\left|\frac{u}{v}\right|<1$

$I[u, v]=\frac{i}{u} \cdot \exp \left(\frac{i}{2} \cdot u\right) \cdot\left[\exp \left(-i \cdot \frac{u^{2}+v^{2}}{2 \cdot u}\right)-\sum_{n=0}^{\infty}\left(\frac{v}{i \cdot u}\right)^{n} \cdot J_{n}(v)\right]$ when $\left|\frac{u}{v}\right|>1$

The complex amplitude is now computed from a sum of Bessel's functions (common software tool), at a precision level (unachievable with optical engineering softwares) set by the number of terms in the sum.

\section{2. calculation of rejection}

The Gaussian complex amplitude at entry ( monomode fiber used as source), is given by: $G_{i n}(\vec{r})=A \cdot \exp \left(-\pi \cdot \frac{r^{2}}{b^{2}}\right) \cdot \exp \left(\frac{i \cdot \pi \cdot r^{2}}{\lambda \cdot \rho}\right)$ and the amplitude coming out of the input-pupilla is $A_{0}(\vec{r})=G_{i n}(\vec{r}) \cdot P_{i n}(\vec{r})$ with $P_{i n}(\vec{r})=\Pi\left(\frac{r}{R_{i n}}\right)$ the classical circular pupilla function with radius $R_{i n}$.

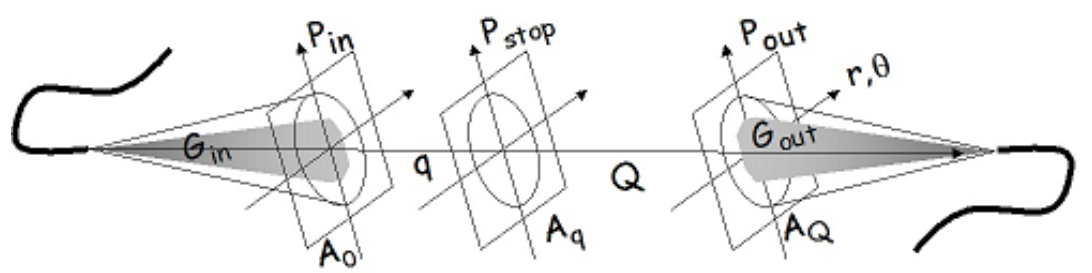

Figure 4. One arm of the bench, with aperture stop on the way from entry to exit. 
Calculation of amplitude $A_{q}$ arriving on the aperture stop at distance "q" from entry is carried as described in the previous section. For writing convenience, now and on, we use the simplified notation $A_{q}=A_{0} * Z_{q}$. The amplitude coming out of the aperture stop (transmission $P_{\text {stop }}$, radius $R_{\text {stop }}$ ) simply is $A_{q} \cdot P_{\text {stop }}$. After propagation along the distance "Q", we find the amplitude $\left[A_{q} \cdot P_{\text {stop }}\right] * Z_{Q}$ and we finally have the amplitude $A_{Q}=\left(\left[A_{q} \cdot P_{\text {stop }}\right] * Z_{Q}\right)$. $P_{\text {out }}$ transmitted by the output pupilla (radius $R_{\text {out }}$, transmission $\left.\Pi\left(\frac{r}{R_{\text {out }}}\right)\right)$.

To calculate the rejection $R e j$, we need to derive the algebraic expression of the amplitude driven into the monomode fiber at exit (serving as spatial filter). This expression is given by the "superposition integral" involving the Gaussian mode $G_{\text {out }}$ propagated by the fiber:

$$
a=\oint_{r<R_{\text {out }}, \theta} A_{Q}(\vec{r}) \cdot G_{\text {out }}(\vec{r}) \cdot r . d r . d \theta
$$

The point now, is that calculation of $A_{Q}$ cannot be carried like was done for $A_{q}$ because the Lommel's approach requires a Gaussian amplitude, what is not the case for $A_{q} . P_{\text {stop }}$. An algebraic derivation pretending to use the expression: $A_{Q}=\left(\left[A_{0} * Z_{q}\right] . P_{\text {stop }}\right) * Z_{Q}$ is quite tricky and tedious.

Fortunately it is possible to use an alternate approach. The amplitude "a" can be a little more explicitly written as:

$$
a=\oint_{r<R_{\text {out }}, \theta}\left[\left(\left[A_{0} * Z_{q}\right] . P_{\text {stop }}\right) * Z_{Q}\right] \cdot\left[G_{\text {out }} . P_{\text {out }}\right]
$$

Using the Parseval-Plancherel relation, we can write:

$$
a=\oint_{r<R_{\text {out }}, \theta}\left[\left(\left[\hat{A}_{0} \cdot \hat{Z}_{q}\right] * \hat{P}_{\text {stop }}\right) \cdot \hat{Z}_{Q}\right] \cdot\left[\hat{G}_{\text {out }} * \hat{G}_{\text {out }}\right]
$$

Now, playing with brackets and products within the integral and applying again the Parseval-Plancherel relation we find the expression:

$$
a=\oint_{r<R_{\text {out }}, \theta}\left(\left[G_{\text {in }} \cdot P_{\text {in }}\right] * Z_{q}\right) \cdot\left(P_{\text {stop }}\right) \cdot\left(Z_{Q} *\left[G_{\text {out }} \cdot P_{\text {out }}\right]\right)=\oint_{r<R_{\text {out }}, \theta} U_{\text {in }} \cdot P_{\text {stop }} \cdot V_{\text {out }}
$$

In the second (compacted) expression are found $U_{\text {in }}$ and $V_{\text {out }}$ which both can be calculated using the Lommel's formalism (they can be seen as given by direct and reverse propagation respectively). Applying this policy to both arms on the bench (CF: ConFocal unit, FF: Flat-Flat unit) we have the collected and residual amplitudes $\left(a_{F F}\right.$ and $\left.a_{C F}\right)$ from which we calculate the rejection:

$$
\text { Rej }=\frac{\text { collected energie }}{\text { residual energie }}=\frac{\left|a_{F F}+a_{C F}\right|^{2}}{\left|a_{F F}-a_{C F}\right|^{2}}
$$

\section{Exemples of expected rejection curves}

Technological constraints, independent of the Focus-Crossing approach have led to split the spectral domain, in two sub-domains. In addition, geometrical constraints prevented to choose the appropriate set of parameters for beams and apertures, which would have provided the required level of rejection over the whole of each spectral interval. Current conditions yields rejection illustrated by the curves in figure 5 , next page. 

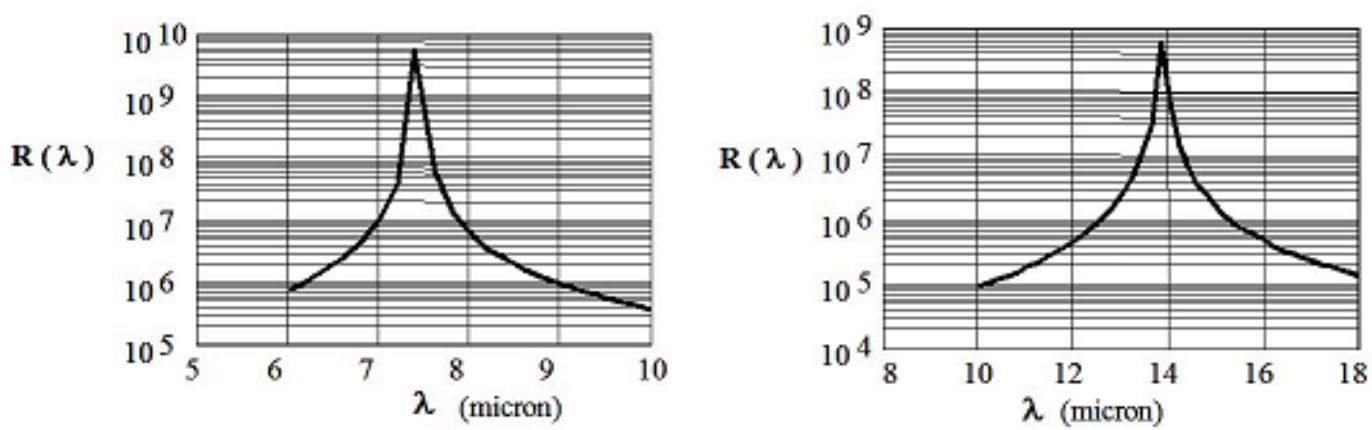

Figure 5. rejection curves obtained with current constraints on bench, for apertures and beam parameters.

\section{Conclusion}

Calculation of rejection in presence of Fresnel's diffraction adverse effect, and with "corrective" aperture stops in suitable locations, has been carried via Lommel's formalism, more reliable and convenient than usual optical engineering softwares in the present context. This approach made appropriate by the use of circular apertures and Gaussian beams can be applied to other set-ups.

\section{References}

Born, M. \& Wolf, E. 1980, Principles of Optics, Pergamon Press

ESA 2000 IRSI-Darwin Report, ESA-SCI (2000) 12

Gouy, C., 1890, C.R. Acad. Sci. Paris, vol 110, p. 1251

Rabbia et al. 2002, Proc. of Genie Workshop, Leiden, the Netherlands, E. Bakker Editor http://www.strw.leidenuniv.nl/ nevec/GENIE/workshop_2002/abstracts/rabbia.html 

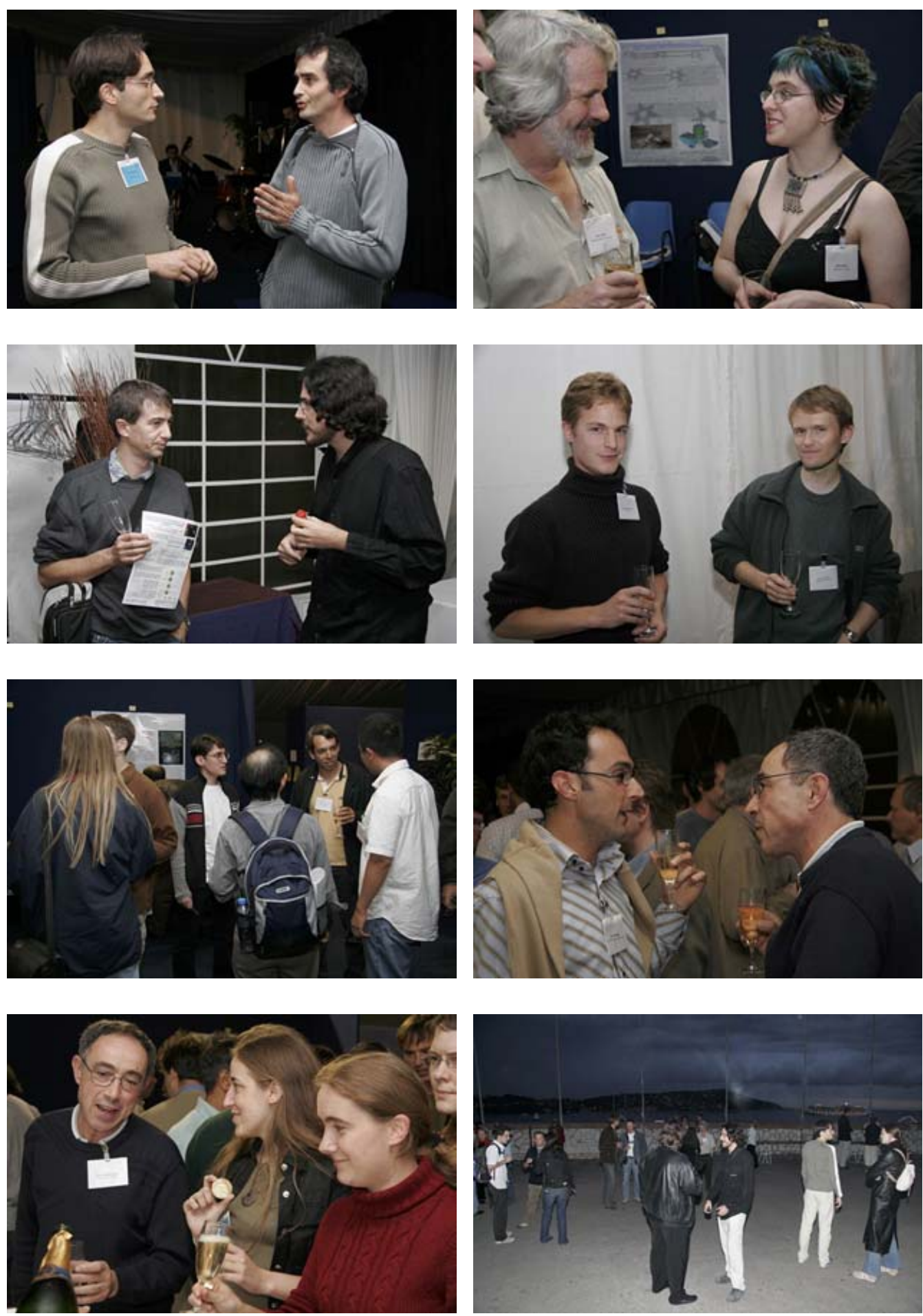

All photographs: Laurent Thareau [1.thareau@free.fr]. 